\title{
A REVIEW OF ANALYTICAL METHODS
}

MEASURING LIPID OXIDATION STATUS IN FOODS: A CHALLENGING TASK

Authors: Blanca Barriuso ${ }^{1}$, Iciar Astiasarán", Diana Ansorena ${ }^{1}(*)$

${ }^{1}$ Department of Nutrition and Food Science, Physiology and Toxicology. Faculty of Pharmacy, University of Navarra, Irunlarrea s/n, 31008-Pamplona, Navarra, Spain. bbarriuso@alumni.unav.es

iastiasa@unav.es

dansorena@unav.es

*Corresponding author: Dr. Diana Ansorena

Mailing address: Department of Nutrition and Food Science and Physiology

Faculty of Pharmacy, University of Navarra, Irunlarrea s/n, 31008-Pamplona, Spain. Tel.: +34948425600 (ext. 6263); Fax: +34948425649.

E-mail address: dansorena@unav.es 


\begin{abstract}
Lipid oxidation analysis in food samples is a relevant topic since compounds generated in the process are related to undesirable sensory and biological effects. As the process is complex and depends on the type of lipid substrate, oxidation agents and environmental factors, proper measurement of lipid oxidation remains a challenging task. A great variety of methodologies have been developed and implemented so far, both for determining primary oxidation products and secondary oxidation products. Most common methods and classical procedures are described, including peroxide value, TBARS analysis and chromatography. Some other methodologies such as chemiluminescence, fluorescence emission, Raman spectroscopy, infrared spectroscopy or magnetic resonance, provide interesting and promising results, so attention must be paid to these alternative techniques in the area of food lipid oxidation analysis.
\end{abstract}

KEYWORDS: Fat oxidation; Hydroperoxides; Secondary lipid oxidation products; TBA; Hexanal 


\section{ABBREVIATIONS}

MDA

PV

AOAC

UV-Vis

TEP

TMP

TBA

TBARs

PAV

HPLC

ESI

MS

$\mathrm{GC}$

DNPH

FID

SOPs

LDI-TOF

HS

SDE

RPDE

SHS

DHS

SPME

CL

IR

FTIR

SERS

NMR

EPR

HPSEC malondialdehyde

peroxides value

association of official analytical chemists

ultraviolet-visible

1,1,3,3-tetraethoxypropane

1,1,3,3-tetramethoxypropane

thiobarbituric acid

thiobarbituric acid reactive substances

para-anisidine value

high perfomance liquid chromatography

electrospray ionization

mass spectrometry

gas chromatography

2,4-dinitro-phenylhydrazine

flame ionization detector

sterol oxidation products

laser desorption/ionization-time of flight

head space

simultaneous distillation extraction

reduced pressure distillation extraction

static head space

dynamic head space

solid phase micro-extraction

chemiluminescence

infrared

fourier transform infrared

surface enhanced Raman spectroscopy

nuclear magnetic resonance

electron paramagnetic resonance

high performance size exclusion chromatography 


\section{INTRODUCTION}

Lipid oxidation in foods constitute a complex chain of reactions that firstly yields primary products (peroxides), that, when exposed to extended oxidation conditions, give rise to secondary oxidation products, including aldehydes, ketones, epoxides, hydroxy compounds, oligomers and polymers. Most of them produce undesirable sensory and biological effects [1, 2]. Therefore, its control is of great importance.

Lipid oxidation occurs via different pathways: radical mechanism (known as autoxidation), singlet oxygen mediated mechanism (known as photooxidation) and also the enzymatic oxidation has been described, catalyzed by lipoxigenases. This review will be focused on the non-enzymatic routes. Both autoxidation and photoxidation give rise to identical or similar peroxides, differing just sometimes in position and stereoisomerism. The first mechanism requires an initial activation energy for the removal of a hydrogen atom, so it is enhanced by high temperatures and presence of double bonds. The latter is triggered by the highly reactive singlet oxygen specie, which is formed by excitation of triplet molecular oxygen, under light exposure and presence of photosensitizers $[3,4]$.

The first compounds formed during oxidation process are peroxides, especially hydroperoxides; hence they are called primary oxidation products. Despite being intermediate compounds of lipid oxidation process, they are relatively stable (depending on the lipid structure), and can be used to assess lipid oxidation status in food samples, providing not too advanced autoxidation is developed in the sample. Because of this intermediate characteristic, temperature conditions during analysis must be controlled to avoid hydroperoxide decomposition, and addition of antioxidant is often required. 
Hydroperoxides usually suffer further oxidation to give secondary oxidation products.

Silvagni et al. [5] proposed an alternative kinetic model where the aldehydes are generated not only via direct degradation of hydroperoxides but from peroxyl radicals through an independent pathway. This mechanism involves a bimolecular reaction to form intermediate tetraoxides, which are unstable at high temperatures and decompose to give alkoxyl radicals. The wide variety of secondary oxidation products to which oxidation gives rise includes aldehydes, ketones, epoxides, hydroxy compounds, oligomers and polymers. Among them, both volatile and non-volatile compounds can be found, such as hexanal or malondialdehyde (MDA), respectively, as main representatives.

Evaluating lipid oxidation status is a challenging task due to a number of reasons. Firstly, different compounds are formed depending on the time, extent of oxidation and mechanism involved. Therefore, choosing just one parameter to analyse the oxidative status is rather difficult and it is frequently more convenient to combine different methods. Besides, as stated by Eymard et al. [6], not only nature and composition of lipid as the substrate of the reaction have an impact on lipid oxidation process, but also type and concentration of proteins, antioxidants and prooxidants present in the food matrix, as well as its physicochemical characteristics. In meat samples, Richards and Dettmann [7] suggested that rates of lipid oxidation may depend on the relative ability of haemoglobins from different animal species to promote it. Chen et al. [8] proposed that colloidal structures formed by phospholipids in vegetable oils could have an impact on the oxidative stability of food oils. Lipid oxidation was observed to be delayed in fish sausages after the addition of several antioxidants [9]. Milk samples oxidation has been recently studied in the presence of catechins and ascorbic acid [10]. On the other hand, each method allows a number of different experimental conditions, and this, together with the lack of uniformity among laboratories, leads to (at least for the moment unavoidable) dissimilar results. Finally, most of the oxidation compounds are prone 
to be further degraded, which provides an added source of divergence. Therefore, a precise control of the experimental procedure must be kept.

Related to lipid oxidation in food samples, other assessments can be also performed. On the one hand, determination of parameters highly indicative of lipid deterioration and subsequent enhanced susceptibility to oxidation (such as hydrolysis of triglycerides) is very common. On the other hand, measuring the time required by a sample to achieve a certain oxidative level through artificially promoting oxidation is another valid procedure to evaluate lipid susceptibility to oxidation (and/or oxidation stability). However, this review will only focus on methods determining the actual and current lipid oxidation of a sample, discarding procedures assessing hydrolytic status and those involving induction of oxidative degradation, since they are not properly indicators of oxidation status but of oxidative susceptibility and stability, respectively.

This review will describe traditional methods to determine both primary and secondary lipid oxidation products in foods, from spectroscopic to chromatographic techniques. Their characteristics, advantages and limitations will be pointed out. Then, alternative methodologies developed during last decades will also be revised in order to provide the complete oversight of possible options. Table 1 summarizes the main characteristics of the methods described in this review.

\section{PRIMARY OXIDATION PRODUCTS}

\subsection{PEROXIDES}

Hydroperoxides redox properties are the base of some of the key methods applied in their determination. A number of reagents can be oxidized by hydroperoxides, including simple inorganic ions, such as iodide or ferrous ion. These methods usually require subsequent complexation to improve the sensitivity. 


\subsubsection{Volumetric method}

Among the different methods proposed for the analysis of peroxides, the iodometry has been the most conventional and widespread method mainly due to the simplicity of the experimental procedure. Although the procedure requires prior lipid extraction, rapid and easily understandable results are provided.

In acidic medium, hydroperoxides and other peroxides react with the iodide ion to generate iodine, which is tittered using a sodium thiosulfate solution, in the presence of starch solution. The AOAC offers an official method since 1965 [11]. According to this method, Peroxide Value (PV) is considered to represent the quantity of active oxygen (in meq) contained in $1 \mathrm{~kg}$ of lipid and which could oxidize potassium iodide.

It shows however some drawbacks, mainly derived from the iodide high susceptibility to oxidation in the presence of molecular oxygen and accelerated by light exposure. Also spontaneous hydroperoxide formation can occur (which would lead to overestimation) and absorption of iodine by unsaturated fatty acids (which would lead to underestimation) [12]. Moreover, it requires anhydrous systems to avoid interference problems, for what lipid extraction is required, and this procedure stage increases the contact with oxygen. In addition, the Peroxide Value determination does not give a real measure of the oxidative degradation, since peroxides are usually further degraded, so simultaneous measurement of secondary products would be appropriate.

\subsubsection{VIS-UV spectroscopic methods}

As well as the volumetric method, spectroscopic ones are rather simple and are moderately sensitive, reliable, and reproducible when carried out under standardized 
conditions. However, they are highly empirical as they measure complex mixtures of oxidized molecules. In addition, they are generally work-intensive and use large amounts of solvents and reagents that might be hazardous [13].

\subsubsection{Ferrous oxidation method}

The ferrous oxidation method for determination of peroxide content is simpler to use than iodometry. The main reason is the lower sensitivity of ferrous ion to spontaneous oxidation by oxygen in air, as compared to high susceptibility to oxidation of iodide solutions. It consists of oxidation of $\mathrm{Fe}(\mathrm{II})$ to $\mathrm{Fe}(\mathrm{III})$, mediated by hydroperoxide reduction in acidic conditions and in the presence of thiocyanate or xylenol orange (in this later case, method is known as FOX). These two compounds provide the spectrophotometric properties, as they form complexes with the ferric ion, giving maximum absorbance peaks at $500 \mathrm{~nm}$ and $560 \mathrm{~nm}$ respectively, which can be measured with a UV-Vis Spectrophotometer [6, 14-18]. However, neither of the methods is free from complications [19]. The thiocyanate method requires large amounts of solvent, and as for the FOX, it detects in a small range of peroxides concentrations and molar absorptivity of the ferrilxylenol orange complex varies with different procedures of making the dye. Nuchi et al. [20] concluded that FOX results (from degradation of fat for feed uses) correlated better with other oxidation parameters than traditional iodometry.

\subsubsection{Iodide oxidation method}

A spectrophotometric iodide-dependant method has also been set to determine hydroperoxide content. In this methodology, not so commonly used [21], the lipid sample is placed in an acidic solution, which is then merged with iodide. The lipid hydroperoxide oxidises iodide to iodine. Then, generated iodine and iodide (in excess) react to give triiodide anion, which is detected spectrophotometrically at $350 \mathrm{~nm}$. Bloomfield [22] used Fe (II) as a catalyst. The 
closed conditions prevent interference from atmospheric oxygen and the short reaction time minimises interference from side reactions.

\subsubsection{Chromatography}

Methodologies explained up to here are in general quite simple regarding theory base, implementation of the procedure and ulterior interpretation of the data, presenting low to moderate selectivity and sensitivity, though. On the other hand, chromatographic techniques are far more accurate, sensible and specific for the compound in interest, allowing better identification of individual products. Indeed, their implementation for hydroperoxides determination instead of that of volumetric and spectroscopic measurements is growing up more and more over the last years. As an unavoidable consequence, chromatographic methods usually require long or meticulous experimental work, precise control of the experimental conditions and the data processing presents some complexity.

\subsubsection{Liquid chromatography}

High Performance Liquid Chromatography (HPLC) is being recently used to determine hydroperoxides. This method is highly sensitive and pretty versatile considering both column and detector properties, allowing to analyze compounds with different characteristics of volatility, molecular weight or polarity. On the other hand, sample preparation is frequently tedious and usually requires lipid extraction. Zeb and Murkovic [23] found the isocratic HPLC-ESI-MS a useful method for the identification and characterization of oxidized species of triacylglycerols (TAGs), i.e. mono- and bis-hydroperoxides. Gotoh et al. [24] developed a method for measuring the peroxide value in colored lipids on the basis of the reaction with triphenylphosphine, forming a compound which absorbs at 260nm. Sample then underwent HPLC separation and UV detection. Ferrous oxidation mediated methods have also been adapted to HPLC separation [25]. Specific hydroperoxides generated from sterols can also be 
assessed by liquid chromatography. Saynajoki et al. [26] determined stigmasterol hydroperoxides by means of a normal-phase column and two types of detectors (UV and fluorescence).

\subsubsection{Gas chromatography}

Gas chromatography coupled to mass spectrometry (GC-MS) can also be used for the analysis of lipid hydroperoxides, but due to their thermo-lability, previous reduction is needed. This, along with the prior lipid extraction and subsequent derivatization step, makes it a cumbersome and time consuming method [27].

\subsection{CONJUGATED DIENES/TRIENES}

Hydroperoxide formation from polyunsaturated fatty acids is generally (over $90 \%$ of the cases) accompanied by stabilization of the radical state via double-bond rearrangement (electron delocalization), which gives rise to conjugated dienes and trienes. These relatively stable compounds absorb in the UV range ( $235 \mathrm{~nm}$ and $270 \mathrm{~nm}$ respectively) and this absorption can be measured by spectrophotometric techniques to assess oxidation level [2829]. This technique is simple and rapid but not as widespread as determination of peroxides determinations, probably because it can lead to underestimation since oleic acid hydroperoxides, containing less than two double bonds, cannot be detected. On the other hand, overestimation is possible if conjugated double bonds are present in the original fatty acid. Furthermore, it is not suitable for oils that have been heated under conditions that decompose hydroperoxides because interference may occur with absorption of carbonyl compounds [30]. Even so, a number of studies have used them for the monitoring of lipid oxidation during heating treatments, especially in vegetable oils [31-33]. Correlation between $235 \mathrm{~nm}$ absorption values and peroxide values has been reported [34]. 


\section{SECONDARY OXIDATION PRODUCTS}

Lipid primary oxidation products can generate, if submitted to further oxidation conditions, secondary oxidation products, including aldehydes, ketones, epoxides, hydroxy compounds, oligomers and polymers. These compounds show a wide variety of physico-chemical properties, differing mainly in volatility, polarity and molecular weight. Most relevant groups of compounds will be commented (aldehydes, volatiles and polymers), as well as a particular molecule very frequently used as oxidation marker (malondialdehyde).

\subsection{MALONDIALDEHYDE}

Malondialdehyde (MDA) is one of the most abundantly generated aldehydes during secondary lipid oxidation and it is probably the most commonly used as oxidation marker, too.

\subsubsection{UV-Vis Spectroscopy}

The most widely employed method for determination of MDA is the spectrophotometric determination of the red fluorescent MDA-thiobarbituric acid (MDA-TBA) complex.

Reaction occurs by attack of the monoenolic form of MDA on the active methylene groups of TBA, at low $\mathrm{pH}$ and high temperature, giving the mentioned chromophore which offers a maximum absorbance peak at $532 \mathrm{~nm}$. Reaction kinetics depends on the concentration of TBA solution, temperature and $\mathrm{pH}[35]$. Several variations of MDA-TBA method exist, with different procedures currently performed in food analysis: direct heating of the sample, sample distillation, lipid extraction with organic solvents or aqueous acid extraction, followed by acid reaction with TBA. General procedure usually consists of homogenization and centrifugation at acidic medium (usually provided by trichloroacetic acid) and posterior reaction with TBA at high temperatures (around $90-100^{\circ} \mathrm{C}$ ). Nevertheless, there is quite a lot 
of variability in reaction conditions, such as heat treatment exposure time; to illustrate it: Berasategi et al., Peiretti et al., Jung et al. and Jongberg et al. [36-39] left mixture react at boiling water bath for 15, 20, 30 and 40 minutes, respectively. On the other hand, trichloroacetic solution concentrations have also been reported to be different (from $3 \%$ to $15 \% \mathrm{w} / \mathrm{v})$ among works $[9,40]$.

Traditional spectrophotometric TBA test has been criticised for some reasons. Firstly, TBA is not selective to MDA, since it also reacts with many other compounds, such as other aldehydes, carbohydrates, amino acids and nucleic acids [41], interfering in the TBA assay and resulting in considerable overestimation, as well as variability in the results. This is why it is also known as TBA reactive substances method (TBARS). There is also a risk of underestimating the response since malondialdehyde can, under in vivo conditions, form linear or cyclical Schiff bases, or even crosslinked bonds, with lysine and arginine from proteins. So poor quantification sensitivity and poor molecular specificity and selectivity can be attributed to this method. Furthermore, the high temperatures $\left(95-100{ }^{\circ} \mathrm{C}\right)$, extended incubation times and strong acidic conditions commonly required for the reaction of MDA with TBA may cause an artifactual peroxidation of sample constituents even in the presence of added antioxidants. Note finally that malondialdehyde, which is mainly formed from linolenic acid oxidation, does not occur in other oxidized lipids (especially when only one double bond is present, i.e., oleic acid). So, it is often a minor secondary oxidation product, spoiling the role of lipid oxidation marker role usually assumed for this compound.

Despite the mentioned limitations, conventional spectrophotometric MDA-TBA methods are preferred because of their simplicity. In fact, it has been recently suggested as a more accurate and sensitive parameter in assessment of oxidative deterioration than p-anisidine test and hexanal determination [20, 42]. 


\subsubsection{Chromatography}

To overcome some of these limitations, more advanced chromatographic determinations have been developed. These techniques provide, as in the case of hydroperoxides measurement (section 2.1.3) more accuracy, sensitivity and specificity for MDA. Harder experimental work, and certain level of complexity in data processing are the drawbacks.

Some of them [43-48] involve the formation of MDA-TBA complex, purification by chromatography (GC or HPLC) and subsequent detection by MS, UV-Vis or fluorometric detector. And some others use derivatization of MDA instead of reaction with TBA, in order to obtain a detectable compound. Reaction with 2,4-dinitro-phenylhydrazine (DNPH) or pentafluorophenylhydrazine and conversion into pyrazole and hydrazone derivatives are the most commonly used procedures with HPLC separation and spectrophotometric/fluorometric detection [48-50]. On the other hand, conversion into tetramethylacetal or methylpyrazole is more common with GC separation, with Flame Ionization Detector (FID) or Nitrogen/Phosphorus specific detector [50]. Mendes et al. [48] and Marcincak [51] compared two HPLC separation methods for MDA determination (MDA-TBA and MDA-DNPH adduct) with the traditional spectrophotometric MDA-TBA test, in samples of chilled fish and pork. The methods were fast, simple, sensitive and stable and presented overall better performance (based on accuracy, specificity and recovery levels) than the traditional spectrophotometric MDA-TBA test, although MDA-DNPH showed a relatively high limit of detection and a lower reproducibility at lower MDA contents in standards and samples.

\subsection{OTHER SECONDARY OXIDATION COMPOUNDS}

\subsubsection{UV-Vis Spectroscopy}


A number of other aldehydes apart from MDA are generated during lipid secondary oxidation. A spectroscopic method to detect their presence is the p-anisidine value (PAV). It is one of the oldest methods for evaluating secondary lipid oxidation, especially in the analysis of animal fats and vegetable oils. It provides useful information on carbonyl compounds, especially non-volatile $\alpha$-unsaturated aldehydes (such as 2-alkenals and 2,4dienals) because it is based on the reactivity of the aldehyde carbonyl bond on the p-anisidine amine group, leading to the formation of a Schiff base that absorbs at $350 \mathrm{~nm}$. The p-anisidine value is defined as 100 times the absorbance of a solution containing $1 \mathrm{~g}$ of fat in $100 \mathrm{~mL}$ of solvent. It is considered a very simple and rapid methodology. PV and PAV allow calculating total oxidation. This parameter (total oxidation) combines evidence about the past history and present state of an oil, so it allows to estimate the overall extent of oxidation in the food [12].

PAV has been recommended as a good control parameter for secondary oxidation control since it correlates well with peroxides content (FOX and PV), TBA and volatile aldehydes analysis $[20,52]$. In the research field, it has remained a little backward, in favour of other techniques [53].

It is well known that the colorimetric response with p-anisidine varies according to the extent of aldehyde unsaturation. Hence, at identical concentrations, the response is more intense with di-unsaturated aldehydes than with mono-unsaturated aldehydes, which in turn are more sensitive than saturated aldehydes. Moreover, p-anisidine reacts with all aldehydes, irrespective of their origin. This is especially the case for some phenol compounds of virgin olive oil, such as decarboxymethyloleuropeine dialdehyde, which could interfere in the assessment. Finally, studies on correlations between PAV and the organoleptic quality highlighted the efficacy of this test for measuring oxidation in many different lipids. 
However, these correlations may vary markedly between lipids and also according to the prevailing oxidation conditions. Caution is thus required when interpreting this index [28].

\subsubsection{Chromatography}

A number of other compounds apart from carbonyls are generated during lipid secondary oxidation.

Concerning fatty acids, they can suffer oxidation as free form, within triacylglycerols or bonded to phospholipids). Their secondary oxidation products can be assessed by HPLC [54]. However, while this technique may be useful to obtain a fingerprint of the oxidation status of the sample, only a minority of signals can be attributed unequivocally to a specific compound because separation is not good enough. Better quantitative analysis can be carried out by means of GC-FID and GC-MS after derivatization into methyl esters [55]. Development of LDI-TOF-MS and ESI-MS [56-58] has meant a great step forward in this field.

Even though Sterol Oxidation Products (generally known as SOPs) present low levels in foods, they show a number of harmful effects in the organism [59], so a significant number of studies have focused their attention in their analysis. Experimental procedure involves lipid extraction, saponification, purification, derivatization and chromatographic analysis. That determination is challenging in many ways: artifact generation, very low concentrations, matrix effects, incomplete identification and reporting, to note a few [60,61]. GC-MS is the most accurate and commonly applied quantification method for this kind of compounds [6266]. Clariana et al. [67] found this technique better than CG-FID in a study performed with pork meat. Due to the necessity of a derivatization process and the impossibility of analysing thermolabile molecules, some liquid chromatography methods have been recently developed [68-70]. However, liquid chromatography shows lower resolution than gas chromatography, 
and the best way to overcome this problem is coupling it to a mass spectrometer detector, which in this case is quite complex and still has not been well solved. A new fast GC-MS method has been recently developed and applied to cholesterol oxidation products analysis, giving highly promising results [71]. Satisfactory resolution, good repeatability and sensitivity, together with the consequent reduction of the time of analysis and consumables make it a valid alternative to conventional CG-MS.

\subsection{VOLATILES}

Under this group of secondary oxidation products a great diversity of compounds has been included, presenting very different functional groups: aldehydes, ketones, alcohols, short carboxylic acids and hydrocarbons. They all share the property of giving from moderate to high smells and are related to rancidity in sensorial tests. Measurement of these secondary oxidation products is of great importance, since their formation closely relates to the deterioration of flavour. Some of these volatile compounds are highly specific to the oxidative degradation of a particular polyunsaturated fatty acid family: propanal is the main marker of oxidation of n-3 fatty acids, while hexanal and pentanal are markers of oxidation of n-6 fatty acids. Both propanal and hexanal are often used as indicators of lipid oxidation in foods because they can be measured in the sample headspace and their lack of double bonds makes them more stable towards oxidation than unsaturated aldehydes. Nevertheless, hexanal is more frequently measured as its formation is higher than that of most secondary oxidation products, apart from a few exceptions. However, measuring the extent of oxidation with just one or two markers is a rather coarse approach, so methods involving assessment of large set of compounds should be promoted [28].

Gas chromatography is the preferred method to quantify volatile molecules and mass spectrometry detection contributes to identify them. Different methods may be used to 
recover volatile oxidation compounds before chromatographic analysis, including: (a) solvent extraction and (b) headspace (HS) techniques.

(a) Although liquid-liquid extractions are not very suitable to recover the volatile content (because they are long, laborious and require a solvent evaporation step, which leads to substantial volatile compound degradation), novel variants have been recently proposed to overcome some of these limitations. Note especially simultaneous distillation extraction (SDE) and reduced pressure steam distillation extraction (RPDE). Both allow to obtain compounds of relatively high boiling point, but with RPDE evaporation is reached with lower temperatures, avoiding possible artefact formation [72]. SDE and RPDE show the advantage of being able to extract high quantities of target compounds since the volatile fractions generally have high solubility in organic solvents [73,74]. Moreover, Ferhat et al. [75] developed a microwave energy-mediated extraction method. Liquid-liquid extractions are the preferred recovering methods whenever the samples require derivatization step previous to chromatographic analysis (HPLC and GC). DNPH, benzyloxime and thiazolidine derivatives are the most frequently used compounds to improve stability and/or detection by visibleultraviolet spectrometry, flame-ionization, nitrogen-phosphorous and mass spectrometry detection [76].

(b) HS analysis can be performed by static headspace (SHS), dynamic purge-and-trap headspace (DHS) or headspace-solid phase microextraction (HS-SPME) techniques. All of them are prior to gas chromatography analysis.

In SHS method, the sample is placed in an airtight vial. Most compounds that are volatile at the analysis temperature evaporate from the liquid or solid fraction and pass into the overhead gas HS. At equilibrium, an aliquot is harvested and injected on the GC column. This method 
is relatively inexpensive and easy to use, it does not require solvent extraction and can be automated. However, as equilibrium is established between the volatile compounds in the HS and those remaining in the sample, only low quantities of compounds are actually recovered, which limits the sensitivity. The increase in the extraction temperature could increase the volatilization of the target compounds and thus increase the quantities recovered, but the temperature must be kept as low as possible in order to minimize generation of new oxidation products and/or thermal degradation of oxidation markers. A number of authors $[77,78]$ have applied this method in food samples analysis.

On the contrary, DHS technique does not require the establishment of equilibrium: the sample is continually purged by inert gas to extract volatile compounds. Then, the gas effluent passes through a porous polymer trap that collects volatile analytes. Among all available trap materials, tenax is the most commonly used. As volatiles contained in the sample are constantly released and trapped, a high concentration of compounds are injected on the GC column. Despite its high sensitivity, the instrumentation is complex and expensive, thus increasing the sources of error (trap drying, trap transfer, purging efficiency, etc.) and it is in general terms slower than SHS. Nevertheless, several studies have highlighted the efficacy of DHS-GC in assessing the oxidative status of different food matrix $[79,80]$.

In SPME analysis, volatile compounds make a first equilibrium between sample and HS, followed by a second one between the HS and the contact fibre (which is coated with a highly adsorbant polymeric film). Finally, the fibre is introduced in the GC injector. This method provides many advantages over other ones, including easy manipulation and experimental set up, short sampling times, easy automation and high sensitivity [81]. A number of authors have applied this method for food lipid oxidation determinations [80,82]. Its main drawback 
is that fibre degradation and contamination occurs quite rapidly, thus replacement is required periodically.

Recent comparative studies performed with all these methods for capture of volatile content lead to the conclusion that each one presents its shortcomings and advantages $[83,84]$, but HS-SPME is being used to an increasing extent on account of its most promising results.

\subsection{OLIGOMERS/POLYMERS}

During extended oxidation, a lipidic compound can be linked together with other one or several ones, giving rise to dimers, oligomers or polymers. Simultaneous analysis of oxidized forms of triacylglycerols and their oligo/polymers is very common to assess lipid oxidation progress. Monomers are very reactive and highly correlate with peroxide value, so they could give information about the primary oxidation level of a sample. On the contrary, triacylglycerols oligopolymers are rather stable compounds, being considered as good indicators of secondary oxidation status $[85,86]$.

High Pressure Size Exclusion Chromatography (HPSEC) has demonstrated to provide satisfactory results in the analysis of this kind of oxidation products. It allows separation and subsequent identification and quantification of molecules according to their molecular weight. It is usually performed on polar compounds, so it requires a previous purification of the polar lipid fraction, which is usually done by silica gel column chromatography. Some studies [1, 87-88] have demonstrated the usefulness of HPSEC in the determination of the levels of the oxidative degradation of a variety of food samples, and particularly that of refined vegetable oils, whose technological process involves quality deterioration. Morales et al. [33] applied it for the determination of advanced oxidation in vegetable oils through the detection of fatty 
acids polymers. Oligomers formation during thermo-oxidation of phytosterols has also been reported [89-92] by means of HPSEC analysis.

\section{ALTERNATIVE METHODOLOGIES}

The previous techniques are either too empirical or highly dependant on several experimental factors, such as technician skill, light exposure and atmospheric oxygen, apart from the fact of being time-consuming. To avoid these limitations, various methodologies have been proposed as good alternatives in analysis of both primary and secondary oxidation products. They are based on direct spectroscopic analyses of samples, such as magnetic resonance, fluorescence and vibrational spectroscopy, and on chemiluminescent properties. As general good points, preliminary treatment is minimal or unnecessary, low amount of sample is required and highly specific results are obtained.

\subsection{Chemiluminescence}

Certain chemical reactions generate electromagnetic radiation. This emission of energy is known as chemiluminescence (CL) and it can be applied to detect and quantify compounds of interest. However, light intensity is very low (ultraweak CL is accompanied during oxidation of hydrocarbons and lipids [93]), so light amplifiers should be introduced to increase it. One of the most commonly used one is the luminol. The luminol-enhanced chemiluminescence involves oxidation of luminol in basic solution generating a free radical intermediate which reacts with flux of oxidizing agents (active free radicals) present in the system, e.g. lipid hydroperoxides. This leads to formation of luminol derived product in excited state, which eventually returns to ground state emitting strong blue light at $430 \mathrm{~nm}$ [94]. Different versions of this method differ in the type of active free radical produced and 
the way of free radical production as well as in details of the procedure. Robinson et al. [95] suggested the addition of $p$-iodophenol to provide more intensive, prolonged, and stable light emission as compared to the traditional luminol system. More recently, a new chemiluminescence method in non aqueous medium CL was developed to detect lipid peroxides in vegetable oils [96], presenting good correlation with spectrophotometric PV analysis.

Baj et al. [97] discovered that partial exclusion of oxygen from the reaction medium strongly influenced the light intensity of the luminol reaction, and the effect is dependent on the oxidant analyzed, so an alternative mechanism was suggested for some oxidant species. Besides, they stated that the oxygen concentration always affects the reproducibility of the results, so equilibrating the working solutions with oxygen or air should always lead to improved results.

The attractive features of CL methods are their higher quickness (taking only a few minutes), sensitivity (picomol levels have been assessed), low sample requirements, low cost and simplicity as compared with other methods [98]. As for shortcomings of this kind of methods, first of all, the kinetic theory and mechanism for chemical processes resulting in CL is not known in detail. This may mean problems with data interpretation. Furthermore, this method is not specific to the lipids (other oxidizing agents also give signal); but this opportunity can be seized to estimate the overall total oxidant status of the sample.

Bunting and Gray [99] developed an automated flow injection chemiluminescence system for measuring lipid hydroperoxide concentrations in oils and found good agreement with a traditional iodometric titration assay, what could denote the usefulness of CL methods to assess lipid primary oxidation; and also in vegetable oils, Yang et al. [100] found a similar trend for TBARs and CL measurements during oxidation. 


\subsection{Fluorescence spectroscopy}

When a compound is irradiated with an electromagnetic energy source, some of their electrons promote from their fundamental state to an excited one, and subsequently they return to their original state, re-emitting the energy previously absorbed. Nevertheless, certain compounds can lose some of that energy as heat, what allows their electrons to return to a higher level than the original one, so emitted light is in this case lower than the absorbed one. This phenomenon is named as fluorescence, and compounds presenting this property, fluorescents. Beam of light is usually from the UV range and emitted energy is typically, but not necessarily, from the visible range. It can be used in analytical chemistry for both qualitative and quantitative determinations, as well as in isolated and coupled to chromatography equipments.

Regarding food field, its implementation is growing up more and more [101]. The free amino groups of proteins can react with aldehydes from lipid peroxidation or reducing sugars to give Schiff bases. These compounds present a high colour intensity (browning) and characteristic fluorescence spectra (excitation and emission wavelengths, and fluorescence intensity) according to the type of protein and adduct. Although its sensitivity is high, excitation and emission wavelength maxima vary depending on the food sample and the procedure followed. They range from $250 \mathrm{~nm}$ to $500 \mathrm{~nm}$ for excitation, and from $280 \mathrm{~nm}$ to $600 \mathrm{~nm}$ for emission [53, 102-104]. Many authors have used the ability of these Schiff bases to emit fluorescence to monitor thermal oxidative processes, especially in dairy products $[105,106]$, meat $[107,108]$, fish $[109,110]$ and oils [111], but fluorescence methodologies are still poorly documented in food lipid oxidation analysis. Both Gatellier et al. [104] and Nguyen et al. [110] found a high correlation between fluorescent pigments and TBARS of 
meat and fish products, which demonstrated that the interaction between proteins and aldehyde products of lipid oxidation is mainly involved in the production of fluorescent pigments and these are good markers of lipid oxidation.

A different implementation of fluorescent properties was developed by Andersen et al. [112] with a cheese sample. They measured the fluorescence of the photosensitizers involved in the lipid oxidation mechanism of the cheese and used the spectra to successfully predict the content of volatile compounds.

\subsection{Infrared spectroscopy}

Infrared (IR) spectroscopy is also known as a very helpful way to study lipid degradation under oxidative conditions [113], particularly since it is an easy, rapid, economical and nondestructive technology. It is based on the determination of fundamental vibrational transitions of a particular compound and involves the absorption of discrete energy levels from the IR region. These discrete energy levels are characteristic of each of atom-atom linkage, so studying the IR spectrum can provide enough information to find out the nature of the analyzed compound. Mathematical tools, such as Fourier Transform (FT) or chemometric methods, permit data processing. Continuous ageing monitoring can be carried out with this methodology, although for the moment, most of the works have been assessed in discontinuous way. Some advances have recently been performed regarding technological devices [114].

IR has been applied to measure the peroxide value in oxidized lipids [115] and differences were found in the IR spectra of fresh and aged oils [116,117]; so IR spectra can be used to characterize the aging of various edible oils [118-122]. The investigation of the FTIR spectra of the treated oils revealed that the microwave heating of oils [123] caused significant changes in the intensities of their absorption bands and produced no shifts in the position of 
the bands. These changes were attributed to the reduction in 18:2 and 18:3 fatty acids content due to the oxidation.

It has also been used for the analysis of edible oils [124], horse mackerel patties [125] and canned tomato juice [126], in combination with other analytical methods which lead to similar conclusions, and therefore providing marker bands to improve the understanding of chemical changes taking place during processing and storage.

\subsection{Raman spectroscopy}

Raman spectroscopy also detects fundamental vibrational transitions although (contrary to infrared spectroscopy) not by means of direct energy absorption, but through an energy (originated from a UV, visible or IR laser) scattering: promotion to a virtual vibrational state and subsequent relaxation to a fundamental vibrational state different from the original one. Therefore, Raman and IR spectroscopy are complementary techniques and provide complementary structural information about molecules. Actually, only some molecules show Raman scattering properties, and most of them at a very small intensity, so quite sophisticated and expensive optical detection equipments are required. This reduces its practical use to a few cases. Indeed, it is still very sparingly used in the food field, in spite of its interesting characteristics, which include being non-destructive, fast, relatively inexpensive, noninvolving chemical products, requiring very little sample preparation, being highly sensitive to unsaturations and poorly sensitive to water $[127,128]$. Two instrumental methods can be employed with Raman spectroscopy: confocal Raman spectroscopy with a powerful laser in visible range and Fourier Transform Raman spectroscopy. Most of the applications on oils have been performed by the later [129]. However, a portable Raman spectrometer has been recently developed [130], which, on the other hand, shows lower resolution than classic ones. Zhang et al. [130] reported the first proof-of-concept study of surface-enhanced Raman detection of a TBA-MDA adduct using silver nanoparticles as the SERS substrate 
Raman spectroscopy results and oxidation levels were related in lipids extracted from several meat and fish products $[128,132]$. In line with peroxide values rises, Raman spectra data showed an increase in particular bands and regions of the spectra of oils extracted which could be attributed to alterations in lipids structure. Furthermore, Raman spectroscopy could be an alternative to gas chromatographic fatty acids analysis, since it successfully predicted total unsaturation and individual compounds several meat products [133]. Salmon Raman spectra [134] indicated differences in the fat fraction (as well as in protein fraction) in coldsmoked products. Regarding vegetable oils studies, Muik et al. [135] detected formation of aldehydes and conjugated double bond systems, as well as isomerization of cis to trans double bonds. The time dependent intensity changes in certain Raman bands were compared to conventional parameters used to determine the extent of oxidation in oils, such as anisidine value and $K_{270}$, and showed good correlation. El-Abassy et al. [136] assessed fatty acid content in olive oil. Zhang et al. [131] developed a method to determine MDA in a model system by means of this technique. They found that it was selective and specific for MDATBA adducts- in terms of differential spectra and high response- versus adducts formed by TBA and other TBARS different from MDA. Besides, they achieved better sensitivity than in works using UV-Vis or fluorescence detectors. Sometimes, reduction of carotenoids content measured by Raman spectroscopy has been used to monitor lipid oxidation process [137].

Simultaneous analysis of the oxidation of edible oils has been also performed by Infrared and Raman techniques [119]. These techniques led to improved information compared to isolated analysis concerning assignment of peaks, and therefore, compounds formed during oxidation.

\subsection{Magnetic Resonance}


The basis of Nuclear Magnetic Resonance (NMR) relies on the property of certain atoms of absorbing and re-emitting energy in the presence of a strong magnetic field due to the excitation of their atomic nuclei. This energy is at a specific resonance frequency which depends on the strength of the magnetic field and on the magnetic properties of the particular isotope of the atom in study. The energy absorptions of the atomic nuclei are affected by the nuclei of surrounding molecules, which cause small local modifications to the external magnetic field. Promising results are obtained by this alternative methodology considering reliability and specificity of the data since they provide an accurate fingerprint of the sample. It does not require extensive manipulation of the sample, thus preserving molecular integrity, and allowing detection of all the substances present in the sample at the same time. This, in addition to its high sensitivity even in complex matrices, highlights the necessity of improving and spreading its use. However, that is a very expensive methodology and requires special skills to interpret the spectra. The use of ${ }^{1} \mathrm{H}$ and ${ }^{13} \mathrm{C}$ NMR spectroscopy in food, applied by different research groups [138-146], has proved to be very useful in evaluating the oxidative status of the lipid fraction, as well as in providing information on the nature (main functional groups) and concentration of the compounds found (i.e. hydroperoxides, carbonyl compounds and dienes). It is considered a valuable tool for quantification of oxidation of food lipids [147], and good correlation with conventional analysis such as TBA has been reported [148]. Several multi-dimensional NMR techniques have been developed in last years (correlational spectroscopy, nuclear overhauser effect spectroscopy, diffusion-ordered spectroscopy...). They allow a better assignment than the one-dimensional spectra, improving the characterization of food lipid samples $[143,149]$. However, the main difficulty derived from the application of these tools is the high time required for the acquisition.

The basis of Electron Paramagnetic Resonance (EPR) is the same as that of NMR but in this case, energy excites spins of single electrons. So, only molecules presenting single electrons 
(that is, radicals) have EPR spectra. It has been used to detect oxidant intermediate species in food matrices $[150,151]$. However, these radicals show quite short lives unless very low temperatures are guaranteed $[13,151-152]$. In an attempt to avoid this problem, some recently developed methodologies deal with the detection of unstable free radicals. Among them, spin trapping techniques allow the indirect detection of lipid-derived radicals by formation of stable spin adducts that can accumulate in detectable concentrations. This way, both identification and quantification of these intermediates is possible. Traps are not radicalspecific, nevertheless particular traps are considered more or less useful for trapping particular radicals. Compounds such as PBN ( $\alpha$-phenyl-tert-butylnitrone) and DMPO $(5,5-$ dimethyl-1-pyrroline-N-oxide) are frequently used for that purpose $[150,153]$.

Combined application of both methodologies (NMR and EPR) is of great interest. In this sense, Silvagni et al. [5] used them in a study investigating the kinetics of thermally induced lipid peroxidation of peanut oil. The use of EPR allowed them to determine the primary alkyl radicals, and provided an estimation of the radical generation rate; whereas by means of NMR, simultaneously detection of primary and secondary oxidation products was performed, thus allowing a more detailed kinetic investigation.

\section{CONCLUSION}

Different kind of compounds can be used as lipid oxidation markers in food samples, among which hydroperoxides and a variety of aldehydes are the most common ones. Each one of them is indicative of a particular state of oxidation, so choosing just one parameter to analyse the oxidative status is rather difficult and it is frequently more convenient to combine different methods. Therefore, analyst must choose carefully the most adequate for his purpose, taking into consideration the most suitable molecules and experimental conditions 
required in each case. First general decision is whether determining primary or secondary

oxidation compounds, considering mainly the extent of oxidation. Afterwards, precision required and characteristics of the food matrix must be considered to follow one methodology or another. A variety of conventional and alternative methodologies have been developed and implemented. Considering the later, they have been proven to provide interesting and promising results, so attention must be paid to these alternative techniques in the area of food lipid oxidation.

\section{ACKNOWLEDGEMENTS}

We thank the "Programa Consolider-Ingenio 2010 CARNISENUSA CSD 2007-00016", the "Proyecto AGL2008-01099/ALI" (Ministerio de Ciencia e Innovación), and "Plan Investigador de la Universidad de Navarra' (PIUNA) for their contribution to the financial support of this work. B. Barriuso is grateful to "Cátedra Tomás Pascual Sanz- Universidad de Navarra" and to "Asociación de Amigos de la Universidad de Navarra" for the Grants received.

\section{REFERENCES}

1. Marquez-Ruiz G, Holgado F, Garcia-Martinez MC, Dobarganes MC, Garcia-Martinez MC (2007) A direct and fast method to monitor lipid oxidation progress in model fatty acid methyl esters by high-performance size-exclusion chromatography. J Chromatogr 1165(1-2):122-127.

2. Kanner J (2007) Dietary advanced lipid oxidation endproducts are risk factors to human health. Mol Nutr Food Res 51(9):1094-1101.

3. Choe E, Min D (2006) Chemistry and reactions of reactive oxygen species in foods. Crit Rev Food Sci Nutr 46(1):1-22.

4. Min D, Boff J (2002) Chemistry and reaction of singlet oxygen in foods. Comprehensive reviews in food science and food safety 1(2):58-72.

5. Silvagni A, Franco L, Bagno A, Rastrelli F (2010) Thermoinduced lipid oxidation of a culinary oil: a kinetic study of the oxidation products by magnetic resonance spectroscopies. J Phys Chem A 114(37):10059-10065. 
6. Eymard S, Baron CP, Jacobsen C (2009) Oxidation of lipid and protein in horse mackerel (Trachurus trachurus) mince and washed minces during processing and storage. Food Chem 114(1):57-65.

7. Richards M, Dettmann M (2003) Comparative analysis of different hemoglobins: Autoxidation, reaction with peroxide, and lipid oxidation. J Agric Food Chem 51(13):3886-3891.

8. Chen BC, Han A, McClements DJ, Decker EA (2010) Physical structures in soybean oil and theirilmpact on lipid oxidation. J Agric Food Chem 58(22):11993-11999.

9. Maqsood S, Benjakul S, Balange A (2012) Effect of tannic acid and kiam wood extract on lipid oxidation and textural properties of fish emulsion sausages during refrigerated storage. Food Chem 130(2):408-416.

10. Mun Y (2011) Effects of green tea catechin on the lipid oxidation, volatile compound formation, and losses of retinol and alpha-tocopherol in whole milk during light illumination as compared with ascorbic acid. Food Sci Biotechnol 20(5):1425-1434.

11. AOAC (2000) Peroxide Value of Oils and Fats. Official Method 965.33, 41:12

12. Sun Y, Wang W, Chen H, Li C (2011) Autoxidation of unsaturated lipids in food emulsion. Crit Rev Food Sci Nutr 51(5):453-466.

13. Kamal-Eldin A, Min D (2010) Lipid oxidation pathways. AOCS Publishings

14. Shantha N, Decker E (1994) Rapid, sensitive, iron-based spectrophotometric methods for determination of peroxide values of food lipids. J AOAC Int 77(2):421-424.

15. Bou R, Codony R, Tres A, Decker E, Guardicila F, Guardiola F (2008) Determination of hydroperoxides in foods and biological samples by the ferrous oxidation-xylenol orange method: a review of the factors that influence the method's performance. Anal Biochem 377(1):1-15.

16. Verardo V, Ferioli F, Riciputi Y, Lafelice G, Marconi E, Caboni MF (2009) Evaluation of lipid oxidation in spaghetti pasta enriched with long chain n-3 polyunsaturated fatty acids under different storage conditions. Food Chem 114(2):472477.

17. Chotimarkorn C, Silalai N, Chaitanawisuit N (2009) Changes and deterioration of lipid in farmed spotted babylon snail (Babylonia areolata) muscle during iced storage. Food Sci Technol Int 15(5):427-433.

18. Sorensen ADM, Nielsen NS, Hyldig G, Jacobsen C (2010) Influence of emulsifier type on lipid oxidation in fish oil-enriched light mayonnaise. Eur J Lipid Sci Tech 112(9):1012-1023.

19. Nielsen N, Timm-Heinrich M, Jacobsen C (2003) Comparison of wet-chemical methods for determination of lipid hydroperoxides. J Food Lipids 10(1):35-50.

20. Nuchi C, Guardiola F, Bou R, Bondioli P, Della Bella L, Codony R (2009) Assessment of the levels of degradation in fat co-and byproducts for feed uses and their relationships with some lipid composition parameters. J Agric Food Chem 57(5):1952-1959. 
21. Watanabe Y, Hashimoto K, Omori A, Uda Y, Nomura M (2010) Suppressive ability of defatted rice bran against lipid oxidation in cookies containing iron. Biosci Biotech Bioch 74(2):262-265.

22. Bloomfield M (1999) The spectrophotometric determination of hydroperoxide and peroxide in a lipid pharmaceutical product by flow injection analysis. Analyst 124(12):1865-1871.

23. Zeb A, Murkovic M (2010) Characterization of the effects of beta-carotene on the thermal oxidation of triacylglycerols using HPLC-ESI-MS. Eur J Lipid Sci Tech 112(11):1218-1228.

24. Gotoh N, Miyake S, Takei H, Sasaki K, Okuda S (2011) Simple method for measuring the peroxide value in a colored lipid. Food analytical methods 4(4):525-530.

25. Sugino K (1999) Simultaneous determination of different classes of lipid hydroperoxides by high-performance liquid chromatography with post column detection by a ferrous xylenol orange reagent. Biosci Biotechnol Biochem 63:773-775

26. Saynajoki S, Sundberg S, Soupas L, Lampi A, Piironen V (2003) Determination of stigmasterol primary oxidation products by high-performance liquid chromatography. Food Chem 80(3):415-421.

27. Lagarda MJ, Manez JG, Manglano P, Farre R (2003)Lipid hydroperoxides determination in milk-based infant formulae by gas chromatography. Eur J Lipid Sci Tech 105(7):339-345

28. Laguerre M (2007) Evaluation of the ability of antioxidants to counteract lipid oxidation: Existing methods, new trends and challenges. Prog Lipid Res 46(5):244282.

29. Shahidi F, Zhong Y (2005) Lipid oxidation: Measurement methods. In: Shahidi F (ed) Bailey's industrial oil and fat products, Wiley and Sons

30. Frankel EN (1998) Methods to determine extent of oxidation. In: Frankel EN (ed) Lipid oxidation, The Oily Press (USA)

31. Maggio R, Valli E, Bendini A, Toschi T (2011) A spectroscopic and chemometric study of virgin olive oils subjected to thermal stress. Food Chem 127(1):216-221.

32. Karoui I, Dhifi W, Ben Jemia M, Marzouk B (2011) Thermal stability of corn oil flavoured with Thymus capitatus under heating and deep-frying conditions. J Sci Food Agric 91(5):927-933.

33. Morales A, Dobarganes C, Marquez Ruiz G, Velasco J (2010) Quantitation of hydroperoxy-, keto- and hydroxydienes during oxidation of FAMEs from high-linoleic and high-oleic sunflower oils. J Am Oil Chem Soc 87(11):1271-1279.

34. Wanasundura UN, Shahidi F, Jablonski CR (1995) Comparison of standard and NMR methodologies for assessment of oxidative stability of canola and soybean oils. Food Chem 52(3): 249-253

35. Fernandez J, Perez-Alvarez J, Fernandez-Lopez J (1997) Thiobarbituric acid test for monitoring lipid oxidation in meat. Food Chem 59(3):345-353.

36. Berasategi I, Barriuso B, Ansorena D, Astiasarán I (2012) Stability of avocado oil during heating: Comparative study to olive oil. Food Chem 132 (1): 439-446. 
37. Peiretti P, Medana C, Visentin S, Giancotti V, Zunino V (2011) Determination of carnosine, anserine, homocarnosine, pentosidine and thiobarbituric acid reactive substances contents in meat from different animal species. Food Chem 126(4):19391947.

38. Jung S, Han B, Nam K, Ahn D, Lee J (2011) Effect of dietary supplementation of gallic acid and linoleic acid mixture or their synthetic salt on egg quality. Food Chem 129(3):822-829.

39. Jongberg S, Skov S, Torngren M, Skibsted L, Lund M (2011) Effect of white grape extract and modified atmosphere packaging on lipid and protein oxidation in chill stored beef patties. Food Chem 128(2):276-283.

40. Leygonie C, Britz TJ, Hoffman LC (2011) Protein and lipid oxidative stability of fresh ostrich M. Iliofibularis packaged under different modified atmospheric packaging conditions. Food Chem 127(4):1659-1667.

41. Salih A, Smith D, Price J, Dawson L (1987) Modified extraction 2-thiobarbituric acid method for measuring lipid oxidation in poultry. Poult Sci 66(9):1483-1488.

42. Pignoli G, Bou R, Rodriguez-Estrada MT, Decker EA (2009) Suitability of saturated aldehydes as lipid oxidation markers in washed turkey meat. Meat Sci 83(3):412-416.

43. Stalikas C, Konidari C (2001) Analysis of malondialdehyde in biological matrices by capillary gas chromatography with electron-capture detection and mass spectrometry. Anal Biochem 290(1):108-115.

44. Jardine D, Antolovich M, Prenzler P, Robards K (2002) Liquid chromatography-mass spectrometry (LC-MS) investigation of the thiobarbituric acid reactive substances (TBARS) reaction. J Agric Food Chem 50(6):1720-1724.

45. de las Heras A, Schoch A, Gibis M, Fischer A (2003) Comparison of methods for determining malondialdehyde in dry sausage by HPLC and the classic TBA test. European food research and technology 217(2):180-184.

46. Cesa S (2004) Malondialdehyde contents in infant milk formulas. J Agric Food Chem 52(7):2119-2122.

47. Seljeskog E, Hervig T, Mansoor M (2006) A novel HPLC method for the measurement of thiobarbituric acid reactive substances (TBARS). A comparison with a commercially available kit. Clin Biochem 39(9):947-954.

48. Mendes R, Cardoso C, Pestana C (2009) Measurement of malondialdehyde in fish: A comparison study between HPLC methods and the traditional spectrophotometric test. Food Chem 112(4):1038-1045.

49. Mateos R, Lecumberri E, Ramos S, Goya L, Bravo L (2005) Determination of malondialdehyde (MDA) by high-performance liquid chromatography in serum and liver as a biomarker for oxidative stress - Application to a rat model for hypercholesterolemia and evaluation of the effect of diets rich in phenolic antioxidants from fruits. J Chromatogr B 827(1):76-82.

50. Ichinose T, Miller M, Shibamoto T (1989) Gas-Chromatographic analysis of free and bound malonaldehyde in rat-liver homogenates. Lipids 24(10):895-898. 
51. Marcincak S, Sokol J, Turek P, Popelka P, Nagy J (2006) Determination of malondialdehyde in pork meat using solid phase extraction and HPLC. Chemické listy 100 (7): 528-532

52. Tompkins C, Perkins EG (1999) The evaluation of frying oils with the p-anisidine value. J Am Oil Chem Soc 76(8):945-947

53. Poulli K, Mousdis G, Georgious C (2009) Monitoring olive oil oxidation under thermal and UV stress through synchronous fluorescence spectroscopy and classical assays. Food Chem 117(3):499-503.

54. Rovellini P, Cortesi N (2004) Oxidative status of extra virgin olive oils: HPLC evaluation. Ital J Food Sci 16(3):333-342.

55. Aguirre M, Marmesat S, Mendez M, Dobarganes M (2010) Application of hightemperature gas chromatography to the analysis of used frying fats. Grasas Aceites 61(2): 197-202.

56. Schiller J, Suss R, Petkovic M, Arnold K (2002) Thermal stressing of unsaturated vegetable oils: effects analysed by MALDI-TOF mass spectrometry, H-1 and P-31 NMR spectroscopy. Eur Food Res Technol 215(4):282-286.

57. Calvano C, Palmisano F, Zambonin C, Palmisano F, Zambonin C (2005) Laser desorption/ionization time-of-flight mass spectrometry of triacylglycerols in oils. Rapid Commun Mass Sp 19(10):1315-1320.

58. Simas R, Catharino R, Cunha IBS, Cabral E, Barrera Arellano D, Eberlin M, et al. (2010) Instantaneous characterization of vegetable oils via TAG and FFA profiles by easy ambient sonic-spray ionization mass spectrometry. Analyst 135(4):738-744.

59. Otaegui Arrazola A, Menendez Carreno M, Ansorena D, Astiasaran I, MenndezCarreo M, Astiasarn I (2010) Oxysterols: A world to explore. Food Chem Toxicol 48(12):3289-3303.

60. Guardiola F, Bou R, Boatella J, Codony R (2004) Analysis of sterol oxidation products in foods. J AOAC Int 87(2):441-466.

61. Busch T, King A (2009) Artifact generation and monitoring in analysis of cholesterol oxide products. Anal Biochem 388(1):1-14.

62. Johnsson L, Dutta P (2006) Determination of phytosterol oxides in some food products by using an optimized transesterification method. Food Chem 97(4):606-613.

63. Menendez-Carreno M, Ansorena D, Astiasaran I (2008) Stability of sterols in phytosterol-enriched milk under different heating conditions. J Agric Food Chem 12 56(21):9997-10002.

64. Ubhayasekera SJKA, Dutta PC (2009) Sterols and oxidized sterols in feed ingredients obtained from chemical and physical refining processes of fats and oils. J Am Oil Chem Soc 86(6):595-604.

65. Derewiaka D, Obiedzinski M (2010) Cholesterol oxides content in selected animal products determined by GC-MS. Eur J Lipid Sci Tech 112(10):1130-1137. 
66. Xu GH, Sun JL, Liang YT, Yang C, Chen ZY (2011) Interaction of fatty acids with oxidation of cholesterol and beta-sitosterol. Food Chem 124(1):162-170.

67. Clariana M, Diaz I, Sarraga C, Garcia Regueiro J (2011) Comparison of the determination of eight cholesterol oxides in dry-cured shoulder by GC-FID, GC-MS, and GC tandem mass spectrometry. Food Anal Method 4(4):465-474.

68. Kemmo S, Ollilainen V, Lampi AM, Piironen V (2008) Liquid chromatography mass spectrometry for plant sterol oxide determination in complex mixtures. Eur Food Res Technol 226(6):1325-1334.

69. Mazalli M, Bragagnolo N (2009) Increase of cholesterol oxidation and decrease of PUFA as a result of thermal processing and storage in eggs enriched with n-3 fatty acids. J Agric Food Chem 57(11):5028-5034.

70. Matsunaga I, Hakamata H, Sadohara K, Kakiuchi K, Kusu F (2009) Determination of oxysterols in oxidatively modified low-density lipoprotein by semi-micro highperformance liquid chromatography with electrochemical detection. Anal Biochem 393(2):222-228.

71. Cardenia V, Rodriguez-Estrada MT, Baldacci E, Savioli S, Lercker G (2012) Analysis of cholesterol oxidation products by Fast gas chromatography/mass spectrometry. J Sep Sci 35(3):424-430.

72. Varlet V, Prost C, Serot T (2007) Volatile aldehydes in smoked fish: Analysis methods, occurence and mechanisms of formation. Food Chem 105(4):1536-1556.

73. Liu R, Xiong K, Dai X, Wang L, Liu Z, Xue W (2010) The effects of maturity on Chilli pepper volatile components determined by SDE, GC-MS and HPLC. Nat Prod Commun 5(6):985-990.

74. Ning L, Fu-Ping Z, Hai-Tao C, Si-Yuan L, Chen G (2011) Identification of volatile components in Chinese Sinkiang fermented camel milk using SAFE, SDE, and HSSPME-GC/MS Food Chem 129 (3): 1242-1252

75. Ferhat M, Tigrine-Kordjani N, Chemat S, Meklati B, Chemat F (2007) Rapid extraction of volatile compounds using a new simultaneous microwave distillation: Solvent extraction device. Chromatographia 65(3-4):217-222.

76. Varlet V, Prost C, Serot T (2007) New procedure for the study of odour representativeness of aromatic extracts from smoked salmon. Food Chem 100(2):820829.

77. Joaquin HJF, Tolasa S, Oliveira ACM, Lee CM, Lee KH (2008) Effect of milk protein concentrate on lipid oxidation and formation of fishy volatiles in herring mince (Clupea harengus) during frozen storage. J Agric Food Chem 56(1):166-172.

78. Vieira C, Fernandez-Diez A, Mateo J, Bodas R, Soto S, Manso T (2012) Effects of addition of different vegetable oils to lactating dairy ewes' diet on meat quality characteristics of suckling lambs reared on the ewes' milk. Meat Sci 91(3): 277-83

79. Nielsen N, Jacobsen C (2009) Methods for reducing lipid oxidation in fish-oilenriched energy bars. International journal of food science technology 44(8):15361546. 
80. Haahr AM, Jacobsen C (2008) Emulsifier type, metal chelation and $\mathrm{pH}$ affect oxidative stability of n-3-enriched emulsions. European Journal of Lipid Science and Technology 110(10):949-961.

81. Iglesias J, Lois S, Medina I (2007) Development of a solid-phase microextraction method for determination of volatile oxidation compounds in fish oil emulsions. J Chromatogr 1163(1-2):277-287.

82. Iglesias J, Medina I (2008) Solid-phase microextraction method for the determination of volatile compounds associated to oxidation of fish muscle. J Chromatogr A, 1192(1): 9-16

83. Shu J, Sun D, Liu S (2010) Comparative performance of different methods used to collect tomato plant volatiles. Allelopathy journal 26(1):71-82.

84. Prosen H, Kokalj M, Janes D, Kreft S (2010) Comparison of isolation methods for the determination of buckwheat volatile compounds. Food Chem 121(1):298-306.

85. Bilancia M, Caponio F, Sikorska E, Pasqualone A, Summo C (2007) Correlation of triacylglycerol oligopolymers and oxidised triacylglycerols to quality parameters in extra virgin olive oil during storage. Food Res Int 40(7):855-861.

86. Gomes T, Caponio F, Durante V, Summo C, Paradiso VM (2012) The amounts of oxidized and oligopolymeric triacylglycerols in refined olive oil as a function of crude oil oxidative level. Food Sci Technol 45(2):186-190.

87. Summo C, Caponio F, Paradiso V, Pasqualone A, Gomes T, Pasqualone A (2010) Vacuum-packed ripened sausages: evolution of oxidative and hydrolytic degradation of lipid fraction during long-term storage and influence on the sensory properties. Meat Sci 84(1):147-151.

88. Caponio F, Summo C, Bilancia M, Paradiso V, Sikorska E (2011) High performance size-exclusion chromatography analysis of polar compounds applied to refined, mild deodorized, extra virgin olive oils and their blends: An approach to their differentiation. Food Sci Technol 44(8):1726-1730.

89. Struijs K, Lampi A, Ollilainen V, Piironen V (2010) Dimer formation during the thermo-oxidation of stigmasterol. Eur Food Res Technol 231(6):853-863.

90. Menendez-Carreno M, Ansorena D, Astiasaran I, Piironen V, Lampi A (2010) Determination of non-polar and mid-polar monomeric oxidation products of stigmasterol during thermo-oxidation. Food Chem 122(1):277-284.

91. Rudzinska M, Przybylski R, Wasowicz E (2009) Products formed during thermooxidative degradation of phytosterols. J Am Oil Chem Soc 86(7):651-662.

92. Rudzinska M, Przybylski R, Zhao Y, Curtis J (2010) Sitosterol thermo-oxidative degradation leads to the formation of dimers, trimers and oligomers: A study using combined size exclusion chromatography/mass spectrometry. Lipids 45(6):549-558.

93. Navas M, Jimenez A (1996) Review of chemiluminescent methods in food analysis. Food Chem 55(1):7-15.

94. Roginsky V, Lissi EA (2005) Review of methods to determine chain-breaking antioxidant activity in food. Food Chem 92(2):235-254. 
95. Robinson E, Maxwell S, Thorpe G (1997) An investigation of the antioxidant activity of black tea using enhanced chemiluminescence. Free Radic Res 26(3):291-302.

96. Szterk A, Lewicki P (2010) A new chemiluminescence method for detecting lipid peroxides in vegetable oils. J Am Oil Chem Soc 87(4):361-367.

97. Baj S, Krawczyk T, Staszewska K, Krawczyk T (2009) The influence of dioxygen on luminol chemiluminescence. Luminescence 24(5):348-354.

98. Rolewski P, Siger A, Nogala-Kalucka M, Polewski K (2009) Chemiluminescent assay of lipid hydroperoxides quantification in emulsions of fatty acids and oils. Food Res Int 42(1):165-170.

99. Bunting J, Gray D (2003) Development of a flow injection chemiluminescent assay for the quantification of lipid hydroperoxides J Am Oil Chem Soc 80(10):951-955.

100. Yang C, Mandal P, Han K, Fukushima M, Choi K (2010) Capsaicin and tocopherol in red pepper seed oil enhances the thermal oxidative stability during frying. J Food Sci Tech 47(2):162-165.

101. Karoui R, Blecker C (2011) Fluorescence spectroscopy measurement for quality assessment of food systems-a review. Food Bioprocess Tech 4(3):364-386.

102. Tironi V, Tomas M, Anon M (2009) Lipid and protein changes in chilled sea salmon (Pseudopercis semifasciata): effect of previous rosemary extract (Rossmarinus officinalis L.) application. Int J Food Sci Tech 44(6):1254-1262.

103. Elmnasser N, Dalgalarrondo M, Orange N, Bakhrouf A, Haertle T, Federighi M, et al. (2008) Effect of pulsed-light treatment on milk proteins and lipids. J Agric Food Chem 56(6):1984-1991.

104. Gatellier P, Sante-Lhoutellier V, Portanguen S, Kondjoyan A (2009) Use of meat fluorescence emission as a marker of oxidation promoted by cooking. Meat Sci 83(4):651-656.

105. Schamberger G, Labuza T (2007) Effect of green tea flavonoids on Maillard browning in UHT milk. Food Sci Technol 40(8):1410-1417.

106. Dalsgaard T, Sorensen J, Bakman M, Nebel C, Albrechtsen R (2011) Light-induced protein and lipid oxidation in low-fat cheeses: whey proteins as antioxidants. Dairy Sci Technol 91(2):171-183.

107. Gatellier P, Gomez S, Gigaud V, Berri C, Le Bihan-Duval E, Sante-Lhoutellier V (2007) Use of a fluorescence front face technique for measurement of lipid oxidation during refrigerated storage of chicken meat. Meat Sci 76(3):543-547.

108. Chelh I, Gatellier P, Sante-Lhoutellier V (2007) Characterisation of fluorescent Schiff bases formed during oxidation of pig myofibrils. Meat Sci 76(2):210-215.

109. Naseri M, Rezaei M, Moieni S, Hosseini H, Eskandari S (2011) Effects of different filling media on the oxidation and lipid quality of canned silver carp (Hypophthalmichthys molitrix). Int J Food Sci Tech 46(6):1149-1156. 
110. Nguyen MV, Thorarinsdottir KA, Thorkelsson G, Gudmundsdottir A, Arason S (2012) Influences of potassium ferrocyanide on lipid oxidation of salted cod (Gadus morhua) during processing, storage and rehydration. Food Chem 131(4):1322-1331.

111. Barrett A, Porter W, Marando G, Chinachoti P (2011) Effect of various antioxidants , antioxidant levels, and encapsulation on the stability of fish and flaxseed oils: assessment by fluorometric analysis. J Food Process Preserv 35(3):349-358.

112. Andersen CM, Andersen LT, Hansen AM, Skibsted LH, Petersen MA (2008) Wavelength dependence of light-induced lipid oxidation and naturally occurring photosensitizers in cheese. J Agric Food Chem 56(5):1611-1618.

113. Kong F, Singh RP (2011) Advances in instrumental methods to determine food quality deterioration. In: Food and Beverage Stability and Shelf Life p.:381-404.

114. Garcia-Gonzalez D, van de Voort FR (2009) A novel wire mesh "cell" for studying lipid oxidative processes by fourier transform infrared spectroscopy. Appl Spectrosc 63(5):518-527.

115. Guillen M, Goicoechea E, Goicoechea E (2007) Detection of primary and secondary oxidation products by Fourier transform infrared spectroscopy (FTIR) and 1H nuclear magnetic resonance (NMR) in sunflower oil during storage. J Agric Food Chem 55(26):10729-10736.

116. Christy A, Egeberg P, Ostensen E (2003) Simultaneous quantitative determination of isolated trans fatty acids and conjugated linoleic acids in oils and fats by chernometric analysis of the infrared profiles. Vibrational spectroscopy 33(1-2):37-48.

117. Rusak D, Brown L, Martin S (2003) Classification of vegetable oils by principal component analysis of FTIR spectra. J Chem Educ 80(5):541-543.

118. Yang H, Irudayaraj J, Paradkar M (2005) Discriminant analysis of edible oils and fats by FTIR, FT-NIR and FT-Raman spectroscopy. Food Chem 93(1):25-32.

119. Muik B, Lendl B, Molina Diaz A, Valcarcel M, Ayora Canada M, Ayora-Caada M (2007) Two-dimensional correlation spectroscopy and multivariate curve resolution for the study of lipid oxidation in edible oils monitored by FTIR and FT-Raman spectroscopy. Anal Chim Acta 593(1):54-67.

120. Le Dreau Y, Dupuy N, Artaud J, Ollivier D, Kister J (2009) Infrared study of aging of edible oils by oxidative spectroscopic index and MCR-ALS chemometric method. Talanta 77(5):1748-1756.

121. Wang H, Liu F, Yang L, Zu Y, Wang H (2011) Oxidative stability of fish oil supplemented with carnosic acid compared with synthetic antioxidants during longterm storage. Food Chem 128(1):93-99.

122. Beltran A, Ramos M, Grane N, Martin ML, Garrigos MC (2011) Monitoring the oxidation of almond oils by HS-SPME-GC-MS and ATR-FTIR. Application of volatile compounds determination to cultivar authenticity. Food Chem 126(2):603609.

123. Moharam MA, Abbas LM (2010) A study on the effect of microwave heating on the properties of edible oils using FTIR spectroscopy. Afr J Microbiol Res 4(19):19211927. 
124. Belhaj N, Arab Tehrany E, Linder M (2010) Oxidative kinetics of salmon oil in bulk and in nanoemulsion stabilized by marine lecithin. Process Biochem 45(2):187-195.

125. Gimenez B, Gomez-Guillen MC, Perez Mateos M, Montero P, Marquez Ruiz G (2011) Evaluation of lipid oxidation in horse mackerel patties covered with boragecontaining film during frozen storage. Food Chem 124(4):1393-1403.

126. Rubio Diaz D, Santos A, Francis D, Rodriguez Saona L (2010) Carotenoid stability during production and storage of tomato juice made from tomatoes with diverse pigment profiles measured by infrared spectroscopy. J Agric Food Chem 58(15):86928698.

127. Reid L, O'Donnell C, Downey G (2003) Recent technological advances for the determination of food authenticity. Trends Food Sci Tech 2006 17(7):344-353.

128. Herrero A (2008) Raman spectroscopy a promising technique for quality assessment of meat and fish: A review. Food Chem 107(4):1642-1651.

129. Korifi R, Le Dreau Y, Molinet J, Artaud J, Dupuy N (2011) Composition and authentication of virgin olive oil from French PDO regions by chemometric treatment of Raman spectra. J Raman Spectrosc 42(7):1540-1547.

130. Guzman E, Baeten V, Fernandez Pierna J, Garcia Mesa J (2011) Application of lowresolution Raman spectroscopy for the analysis of oxidized olive oil. Food control 22(12):2036-2040.

131. Zhang D, Haputhanthri R, Ansar S, Vangala K, De Silva H, Sygula A, et al. (2010) Ultrasensitive detection of malondialdehyde with surface-enhanced Raman spectroscopy. Anal Bioanal Chem 398(7-8):3193-3201.

132. Sarkardei S, Howell N (2007) The effects of freeze-drying and storage on the FTRaman spectra of Atlantic mackerel (Scomber scombrus) and horse mackerel (Trachurus trachurus). Food Chem 103(1):62-70.

133. Beattie JR, Bell S, Borgaard C, Fearon A, Moss B (2006) Prediction of adipose tissue composition using Raman spectroscopy: average properties and individual fatty acids. Lipids 41(3):287-294.

134. Herrero AM, Carmona P, Ordonez JA, Cambero MI (2009) Raman spectroscopic study of electron-beam irradiated cold-smoked salmon. Food Res Int 42(1):216-220.

135. Muik B, Lendl B, Molina Diaz A, Ayora Canada M, Lendl B, Molina-Daz A, et al. (2005) Direct monitoring of lipid oxidation in edible oils by Fourier transform Raman spectroscopy. Chem Phys Lipids 134(2):173-182.

136. El-Abassy R, Donfack P, Materny A (2009) Rapid Determination of Free Fatty Acid in Extra Virgin Olive Oil by Raman Spectroscopy and Multivariate Analysis. J Am Oil Chem Soc 86(6):507-511.

137. Kathirvel P, Ermakov IV, Gellermann W, Mai J, Richards MP (2008) Resonance Raman monitoring of lipid oxidation in muscle foods. Int J Food Sci Tech 43(11):2095-2099.

138. Guillen MD, Ruiz A (2008) Monitoring of heat-induced degradation of edible oils by proton NMR. Eur J Lipid Sci Tech 110(1):52-60. 
139. El Hajjouji H, Merlina G, Pinelli E, Winterton P, Revel J, Hafidi M (2008) C-13 NMR study of the effect of aerobic treatment of olive mill wastewater (OMW) on its lipid-free content. J Hazard Mater 154(1-3):927-932.

140. Dybvik A, Falch E, Rustad T (2008) Solid phase extraction as a tool to separate lipid classes and study deterioration of marine lipids. J Aquat Food Prod Technol 17(1):3959.

141. Tyl C, Brecker L, Wagner K (2008) H-1 NMR spectroscopy as tool to follow changes in the fatty acids of fish oils. Eur J Lipid Sci Tech 110(2):141-148.

142. Colzato M, Scramin JA, Forato LA, Colnago LA (2011) 1H NMR investigation of oil oxidation in macadamia nuts coated with zein-based films. J Food Process Preserv 35(6):790-796.

143. Scano P, Anedda R, Melis MP, Dessi MA, Lai A (2011) (1)H- and (13)C-NMR Characterization of the molecular components of the lipid fraction of pecorino Sardo Cheese. J Am Oil Chem Soc 88(9):1305-1316.

144. Guillen MD, Uriarte PS (2012) Monitoring by $1 \mathrm{H}$ nuclear magnetic resonance of the changes in the composition of virgin linseed oil heated at frying temperature. Comparison with the evolution of other edible oils. Food control 28(1): 59-68

145. Guillen MD, Uriarte PS (2012) Simultaneous control of the evolution of the percentage in weight of polar compounds, iodine value, acyl groups proportions and aldehydes concentrations in sunflower oil submitted to frying temperature in an industrial fryer. Food control 24(1): 50-56

146. Alonso-Salces RM, Holland MV, Guillou C (2011) 1H-NMR fingerprinting to evaluate the stability of olive oil. Food control 22(12): 2041-2046

147. Namal Senanayake SPJ, Shahidi F (2007) Measuring oxidative stability of structured lipids by proton nuclear magnetic resonance. J Food Lipids 14(3): 217-231

148. de Oliveira Silva AC, Marsico E, Guimaraes C, Sloboda Cortez M (2011) Effect of Gamma Radiation on Lipids by the TBARS and NMR. Braz Arch Biol Techn, 54(6): 1343-1348

149. Hatzakis E, Agiomyrgianaki A, Kostidis S, Dais P (2011) High-Resolution NMR Spectroscopy: An Alternative Fast Tool for Qualitative and Quantitative Analysis of Diacylglycerol (DAG) Oil. J Am Oil Chem Soc 88(11): 1695-1708

150. Szterk A, Stefaniuk I, Waszkiewicz Robak B, Roszko M (2011) Oxidative stability of lipids by means of EPR spectroscopy and chemiluminescence. J Am Oil Chem Soc 88(5):611-618.

151. Huvaere K, Nielsen J, Bakman M, Hammershoj M, Skibsted L, Hammershj M, et al. (2011) Antioxidant properties of green tea extract protect reduced fat soft cheese against oxidation induced by light exposure. J Agric Food Chem 59(16):8718-8723.

152. Geoffroy M, Lambelet P, Richert P (2000) Role of hydroxyl radicals and singlet oxygen in the formation of primary radicals in unsaturated lipids: a solid state electron paramagnetic resonance study. J Agric Food Chem 48(4):974-8 
153. Papadimitriou V, Sotiroudis TG, Xenakis A, Sofikiti N, Stavyiannoudaki V, Chaniotakis NA (2006) Oxidative stability and radical scavenging activity of extra virgin olive oils: an electron paramagnetic resonance spectroscopy study. Anal Chim Acta 573: 453-458 